\title{
Parallel, multi frequency EIT measurement, suitable for recording impedance changes during epilepsy
}

\author{
T. Dowrick ${ }^{1,2}$, G. Sato Dos Santos ${ }^{1}$, A. Vongerichten ${ }^{1}$ and D. Holder ${ }^{1}$ \\ 1. Department of Medical Physics \& Biomedical Engineering, University College London, United Kingom. \\ 2.E-mail any correspondence to: t.dowrick@ucl.ac.uk
}

\begin{abstract}
Electrical Impedance Tomography (EIT) has been proposed as a method for imaging and localising epileptic activity in the brain. No existing EIT system meets all of the requirements for effective imaging of epilepsy. A parallel EIT system, employing frequency division multiplexing, is described, which is optimised for measuring impedance changes during epilepsy. The system is capable of imaging short duration, spontaneous events in a saline filled tank, using as little as $1 \mathrm{~ms}$ of recorded data. In-vivo impedance measurements recorded during epilepsy in a rat model are presented.
\end{abstract}

Keywords: Electrical Impedance Tomography (EIT), Biomedical Circuits, Epilepsy

\section{Introduction}

Electrical Impedance Tomography (EIT) is an imaging technique in which differences in impedance are used to produce an image of the internal structure of an object [1]. To date, the most successful clinical implementations of EIT have been in imaging the organs of the torso, including lung [2-4], liver [5,6] and breast imaging [7].

In addition, EIT has been proposed as a method for imaging neural activity [8,9]. One particular application that has shown promise is the imaging of impedance changes during epileptic activity, with an emphasis on seizure focus localisation [10,11], using electrodes placed on the surface of the brain. Epilepsy is one of the most common neurological condition in the world, affecting approximately 50 million people worldwide [12]. In patients who do not respond to anti-epileptic drugs, surgical intervention may be possible to remove the affected portion of the brain [13]. Accurate localisation of the seizure focus is essential and may lead to better post-operative outcomes. Existing work on head EIT has demonstrated that 3D images can be produced [14], with time and spatial resolution of $2 \mathrm{~ms}$ and $200 \mu \mathrm{m}$ in the rat cortex, which has been modelled to translate to an accuracy of $5 \mathrm{~mm}$ in human brain.

Existing methods for seizure localisation include the electrocorticogram (ECoG) [13], where an electrode mat is placed directly onto the surface of the brain, spike triggered fMRI [15] and intrinsic optical imaging [16-19] and it is hoped that EIT can be shown to be a useful addition to this field. Before clinical evaluation of EIT as an effective method for seizure localisation can be carried out, suitable hardware is required, which is tailored to the particular requirements of the application.

\section{Hardware Requirements \& Existing EIT Systems}

A typical EIT system consists of an electrode array, a current source, and a voltage measurement unit. The electrode array is connected to the object to be imaged and a sinusoidal current, of a defined amplitude and frequency, is injected through a pair of electrodes, while voltages are measured at each of the remaining electrodes. This process is repeated for a number of different pairs of injecting electrodes, yielding an array of voltage measurements which can be used to reconstruct the conductivity profile of the target object. EIT systems may inject through a single electrode pair, or multiple electrode pairs simultaneously, at frequencies from $10 \mathrm{~Hz}-1 \mathrm{MHz}$ and typically have 16-32 electrodes. Voltage acquisition at each electrode is performed either serially or in parallel, and frame acquisition times are in the range $10 \mathrm{~ms}-5$ minutes. Existing systems include fEITER [20] (32 electrodes, parallel record, single pair injection at $10 \mathrm{kHz}, 100$ frames/s), KHU [21] (16 - 64 electrodes, parallel voltage record, multiple pair injection, $11 \mathrm{~Hz}-500 \mathrm{kHz}, 100$ frames/s) and Swisstom Pioneer Set (32 electrodes, single pair injection at $50 \mathrm{kHz}, 50$ frames/s). Previous attempts to image 3D epileptic activity employed the UCLH Mark 1b system [22] a 31 electrode system, with serial data record and an injection current of $38.4 \mathrm{kHz}$. However, it was not possible to capture reproducible impedance changes during seizures using this approach, due to the limitations of the hardware.

In epilepsy, there are two types of abnormal activity. Seizures usually comprise continuous epileptic spiking which lasts for up to a minute or so. Each spike is caused by a depolarization lasting up to $70 \mathrm{~ms}$ due to the opening of ion channels and causes a surface negative potential. Within a few seconds, there are movements of water from the extracellular to the intracellular space, as brain cells run out of energy to control their osmotic pressure. These are termed 'ictal' events. Between seizures, there may be frequent individual spike discharges, perhaps every minute or so, which are termed 'interictal' spikes and are not usually noticed by the subject. The impedance change associated with the seizure has two components. For each 


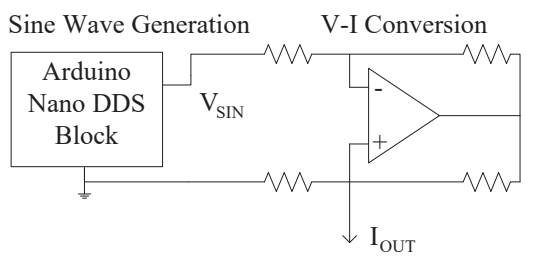

$\mathbf{A}$

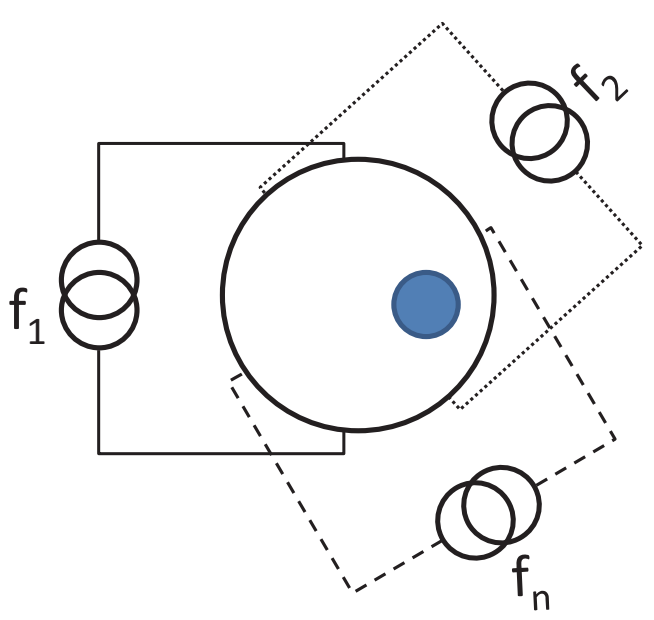

B

Fig. 1: A - Current source implementation. Sine wave generation performed by Arduino Nano with output filtering. Howland current pump used for V-I conversion. B - Example connection of multiple current sources to phantom.

individual spike, the cell depolarisation causes an impedance decrease of approximately $1 \%$ at DC. At measurement frequencies below $\sim 10 \mathrm{kHz}$, the applied current will predominately flow through the extracellular space, but as ion channels open at the seizure onset, some current will flow through the intracellular space, reducing the magnitude of the impedance change to about $0.1 \%$ [23]. Following this initial decrease in impedance, there is an increase of impedance due to cell swelling of up to $10 \%$, lasting several seconds [24].

In order to correlate impedance changes to epileptic activity, it is necessary to record EIT data simultaneously with EEG. Systems, such as the UCLH Mark 1b, which employ serial data record, are not ideally suited to capturing these types of events, as it is necessary to switch between the measurement electrodes. This reduces the temporal resolution of the measured impedance signal, while also introducing a switching artefact into the EEG recording. Switching of the injecting electrodes introduces an artefact which further compounds these problems. The contamination of the EEG signal occurs in all of the EIT systems previously mentioned. While it is possible to remove some of the switching artefact [25], this requires the use of separate electrodes for recording EIT and EEG data, and does not entirely eliminate the problem.
Switching of the recoding electrodes can be eliminated by employing parallel voltage recordings, and several systems have been reported with this capability, including the SwissTom and KHU systems. Overcoming the need to switch the injecting electrodes is more difficult, requiring some form of multiplexing of the input signal. Various implementations have been reported, including frequency division multiplexing (FDM) [26,27] where current is injected simultaneously through multiple electrode pairs, at different frequencies; and code division multiplexing (CDM) [28], where each injected current is modulated by a unique binary code, allowing for multiple simultaneous injections at the same frequency.

Two approaches to imaging seizures can be undertaken. The first is to measure the slow impedance change over several seconds for a single seizure. The second, more difficult approach is to measure the fast impedance change of each individual spike. This approach is more demanding, as a finer time resolution, of several milliseconds, is required. Additionally, the impedance change, of less than $1 \%$, is significantly smaller than for the slow component, making it more difficult to detect. Other applications of neural EIT, such as the imaging of evoked potentials (EPs), can take advantage of averaging multiple identical events together to increase the signal strength. The use of averaging is more difficult in the case of epilepsy, as spiking events occur unpredictably and can vary in strength and number, reducing the uniformity required for averaging. Additionally, some patients display more than one seizure type and it would be advantageous to image each separately.

The choice of operating frequency, bandwidth and number of electrodes associated with the EIT system are also important factors. For neural events, the optimal measurement frequency has been reported to be $\sim 2 \mathrm{kHz}$ [11]. If only the slow component of impedance change is considered, a bandwidth of several $\mathrm{Hz}$ would be sufficient. For the fast changes, occurring over several milliseconds, the signal bandwidth needs to be $>200 \mathrm{~Hz}$ to capture all necessary information. No conclusive data is available on the optimal number of electrodes required, but for existing studies involving 3D neural imaging, 60 is the minimum number of electrodes used [14].

In principle, several of the EIT system described above are able to record the slow impedance changes, but they lack the capability to effectively record the EEG signal accurately. With regard to recording the fast changes, none of the systems have a small enough time resolution, without resorting to averaging, due to the need to switch between injection/recording electrodes. For imaging with EIT in epilepsy, modifications to EIT hardware and data collection are required to achieve the joint goals of improved temporal resolution and avoidance of EEG artefact. We propose that this can be achieved through a number of modifications: frequency division multiplexed injections, in the $\mathrm{kHz}$ range, parallel voltage recording, support for at least 60 electrodes and simultaneous recording of EEG data. 


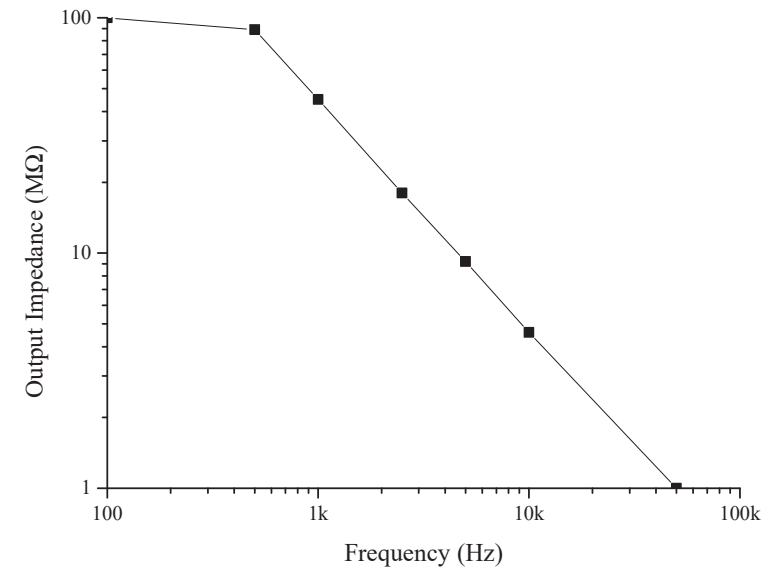

Fig. 2: Output impedance of current source. Measured using HP43284A Impedance Analyser.

The purpose of this work is to describe the construction and testing of such a system. Experiments in a saline filled tank verify the operation of the system for imaging short duration events of several milliseconds, and preliminary data from rat experiments verifies that the system meets the requirements in a practical experimental setting.

\section{Hardware}

A simple AC current source can be constructed from two subcircuits. Firstly, a suitable sinusoidal voltage waveform must be produced. While this can be achieved using purely analog circuitry, such as a Wien Bridge or Quadrature oscillator, more modern approaches utilise some form of direct digital synthesis (DDS) to produce the waveform $[20,21]$. The second stage is a voltage to current converter (V-I), which takes the voltage output of the DDS stage, and converts it into a current. Here, a basic Howland current pump is used which provides a high output impedance and stability across a range of loads and frequencies. An improved HCP circuit is possible [29], but not required.

As no system currently exists that offers independent parallel current injection at closely spaced frequencies, a prototype system was constructed (Figure 1). Sine wave generation was achieved using a form of DDS, implemented on an Arduino Nano, with voltage to current conversion performed using a Howland current pump, made up of an operational amplifier (AD8039) and $10 \mathrm{k} \Omega$, $0.1 \%$ tolerance resistors. A $5 \mathrm{~V}$ battery was used as power supply.

Data recording was achieved through the use of an EEG amplifier. The BioSemi system offers simultaneous sampling of up to 128 channels with a sampler rate of 16 $\mathrm{kHz}$. The bandwidth of the system is $3 \mathrm{kHz}$ ( $-3 \mathrm{~dB}$ point). If a greater range of frequencies are required, other systems (g.tec actiCHamp) are available which can sample up to 40 $\mathrm{kHz}$.

Several key output characteristics of the current source were measured experimentally. The output impedance was measured using a HP4284A, between $100 \mathrm{~Hz}$ and $50 \mathrm{kHz}$. The results are plotted in Figure 2. At the lowest frequency,

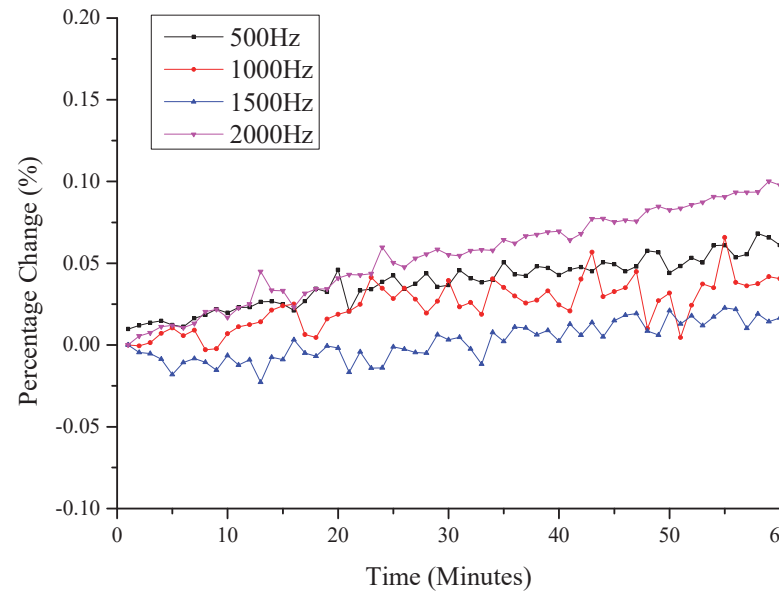

Fig. 3: Stability of current source over time, measured on a resistor phantom. The slight drift over time is thought be caused by device heating.

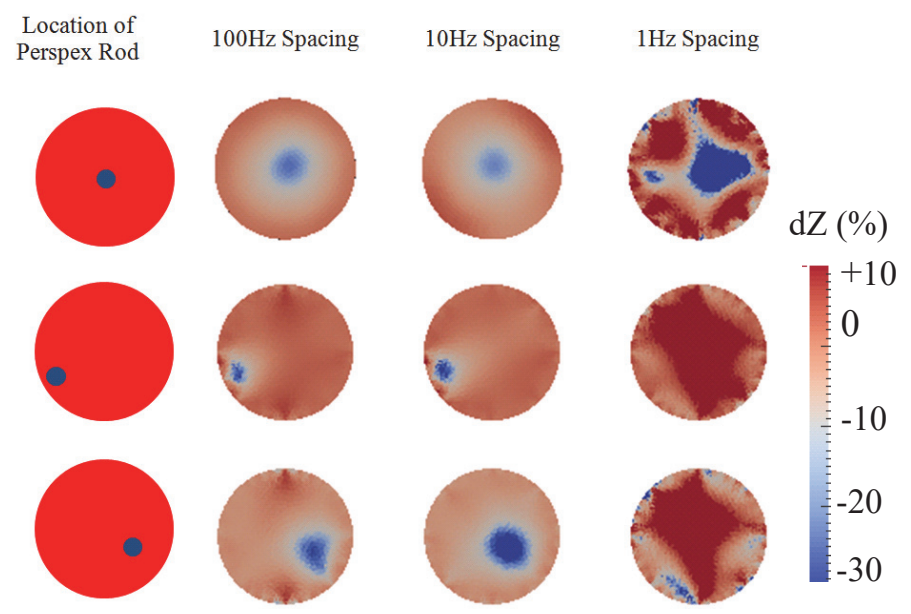

Fig. 4: Results of imaging test. Blue areas represent an increase in impedance. The first column indicates the position of the perturbation in the tank. The remaining columns show the reconstructed images for each frequency group used. Colour bar indicates percentage change in impedance.

the output impedance is nearly $100 \mathrm{M} \Omega$, reducing to $4 \mathrm{M} \Omega$ at $10 \mathrm{kHz}$ and $1 \mathrm{M} \Omega$ at $50 \mathrm{kHz}$.

The amplitude stability of the output current was investigated by connecting four current sources in parallel to a resistor phantom. With each current source injecting at a different frequency $(500 \mathrm{~Hz}, 1000 \mathrm{~Hz}, 1500 \mathrm{~Hz}$, and 2000 $\mathrm{Hz}$ ), voltages were recorded for 1 hour using the BioSemi. The signal at each frequency was extracted in MATLAB (Figure 3 ). The largest change seen is $0.1 \%$, equivalent to a change of $50 \mathrm{nA}$. The average change each minute is less than $1 / 1000$ of a percent $(<0.5 \mathrm{nA})$.

\section{Experimental Setup and Results}

\section{Saline Tank Experiments}

To evaluate the parallel, multi frequency approach to EIT imaging, time difference experiments were produced using a saline filled tank ( $0.9 \%$ saline $)$. Four independent current sources were assembled. The saline tank had 32 electrodes uniformly spaced in a circle around the center. The current 


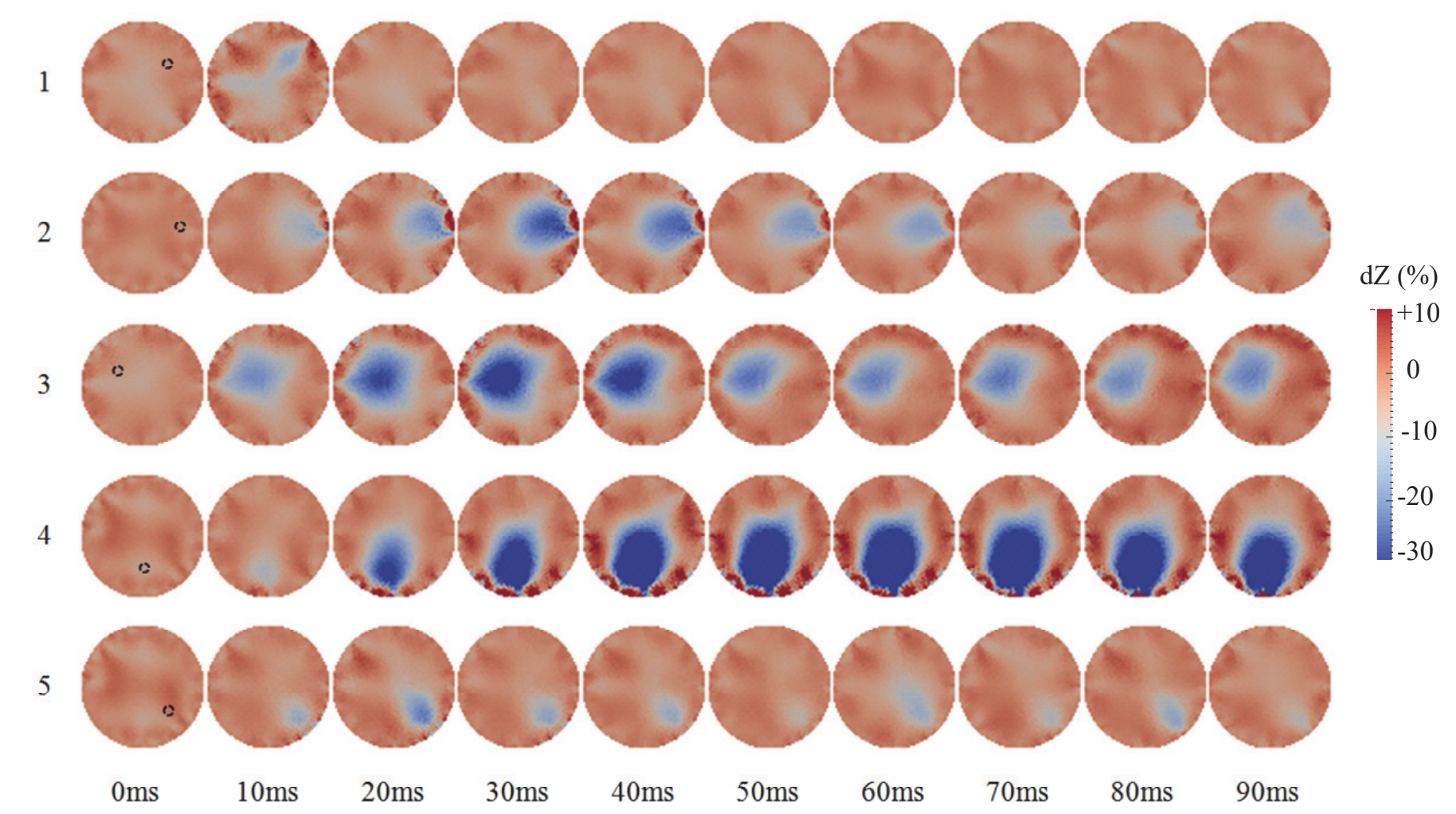

Fig 5: Results of coin drop experiment. 10 frames are shown, taken $10 \mathrm{~ms}$ apart, for each coin dropped. Each frame uses $1 \mathrm{~ms}$ of recorded data. The black circle at $0 \mathrm{~ms}$ indicates the position at which the coin was dropped.

sources were connected to inject between opposite electrodes (1-17, 5-21, 9-25, and 13-29). The contact impedance of each electrode was less than $2 \mathrm{k} \Omega$ at $1 \mathrm{kHz}$. All 32 electrodes were connected to the BioSemi EEG recording system.

Three illustrative frequency groupings were chosen, with spacing between frequencies of $100 \mathrm{~Hz}, 10 \mathrm{~Hz}$ and 1 $\mathrm{Hz}$ (Table 1).

Table 1 - List of frequency groups

\begin{tabular}{|l|l|l|l|l|}
\hline Group A & $1800 \mathrm{~Hz}$ & $1900 \mathrm{~Hz}$ & $2000 \mathrm{~Hz}$ & $2100 \mathrm{~Hz}$ \\
\hline Group B & $1980 \mathrm{~Hz}$ & $1990 \mathrm{~Hz}$ & $2000 \mathrm{~Hz}$ & $2010 \mathrm{~Hz}$ \\
\hline Group C & $1998 \mathrm{~Hz}$ & $1999 \mathrm{~Hz}$ & $2000 \mathrm{~Hz}$ & $2001 \mathrm{~Hz}$ \\
\hline
\end{tabular}

For each frequency configuration, a baseline recording of the tank was taken. Following this, a Perspex rod, $2 \mathrm{~cm}$ in diameter, was introduced into the saline tank as a perturbation, at three separate locations - the centre of the tank, halfway between the centre and electrode 20 and next to electrode 31 .

Data processing and image reconstruction were carried out in MATLAB. For each of the four frequencies used, the data was bandpass filtered and demodulated, producing a set of voltages at each of the 32 electrodes. Two filter designs were considered. The first used a narrow bandwidth $\left(1 / 10^{\text {th }}\right.$ of the frequency spacing). The second used a bandwidth equal to the frequency spacing $(200 \mathrm{~Hz})$ with notch filters used at each of the unwanted injected frequencies, which gives the maximum possible temporal resolution. The difference between the two sets of voltages was far less than the average level of noise seen in the system and can be said to be insignificant. As such, either approach to filtering can be implemented.

Difference data was produced by subtracting the baseline voltages from the voltages obtained in the presence of the Perspex rod. Image reconstruction was performed using a $0^{\text {th }}$ order Tikhonov regularisation [30], on a 60,000 finite element mesh of the cylindrical tank.

Frequency groups A\&B (100 Hz and $10 \mathrm{~Hz}$ spacing) resulted in successful reconstruction of the location of the Perspex rod, accurate to within $4 \mathrm{~mm}$. Group C, with $1 \mathrm{~Hz}$ spacing between frequencies, did not result in acceptable images (Figure 4). In practice, such narrow spacing will not be required. As previously discussed, the required bandwidth will be dictated by the time resolution needed to image the fast impedance change, with a minimum of 200 $\mathrm{Hz}$ required.

These results verify that the hardware operates correctly, but represent a simple test condition, as only static objects are considered. The results are comparable to those produced by existing EIT systems. In order to test the operation of the system under more realistic conditions, comparable to those associated with seizure localisation, an additional experiment was performed. In this second experiment, coins were dropped into the saline filled tank. A coin was chosen for convenience, but for more statistically robust results, other objects, such as a ball bearing could also have been chosen. Each coin disturbed the saline as it fell to the bottom of the tank, leaving behind 
$\mathbf{A}$

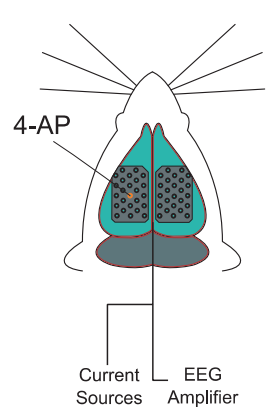

B

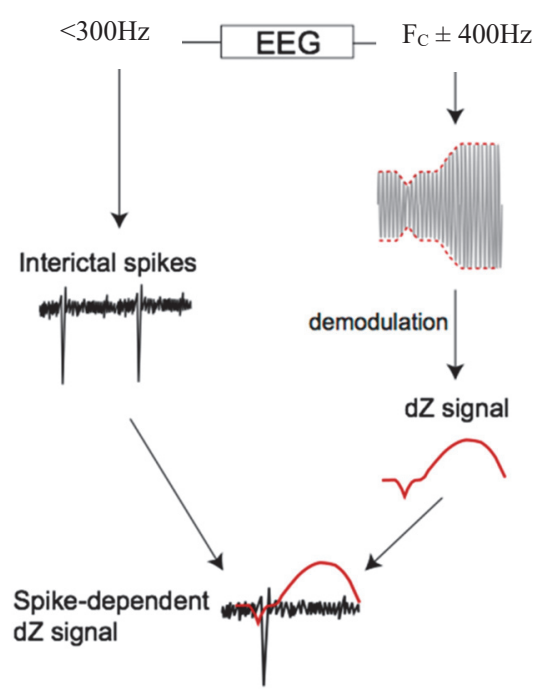

Fig. 6: (A) Experimental Setup. 60 electrodes were placed on either side of the brain. Seizures were triggered using a 4-AP Model. (B) Data extraction. By low pass (EEG) and bandpass (EIT) filtering, the data was split into the EEG component and the EIT component, from which the impedance change was extracted.

an air pocket which persisted for somewhere in the region of 10-100 ms. Imaging the effects of the coins poses a greater challenge, as the durations of the events are relatively short and the exact nature of the air pocket produced is highly variable. In particular, it is dependent on the speed and angle at which the coin breaks the surface of the saline.

The same experimental setup and imaging method was used, with four current sources injecting at 15001800 , 2100 , and $2400 \mathrm{~Hz}$. A total of five coins were dropped into the tank at different locations, one every 30 seconds. By splitting the recorded data into $1 \mathrm{~ms}$ frames, and imaging each frame with reference to the first frame, it was possible to reconstruct the locations at which the coins were dropped and the subsequent disturbance of the saline.

There were noticeable variations in magnitude and duration of the disturbance of the saline, corresponding to the varying trajectories of the coins. There is a clear relationship between the size of the perturbation created and its duration. The effect of the first coin is both the shortest and smallest $(10 \mathrm{~ms})$, while the fourth coin causes the largest and longest lasting disturbance ( $>100 \mathrm{~ms})$ (Figure 5). This is as expected; larger air pockets will naturally take longer to form and disperse.

\section{In-vivo impedance measurements during epileptic seizures}

Having verified the functionality of the hardware through tank experiments, preliminary work was undertaken to test the system in an animal model, to measure the slow impedance change during epilepsy in rats [11,31] (Figure 6A). A female Sprague-Dawley rat was anaesthetised with isoflourane, and maintained with $\alpha$-chloralose and fentanyl.
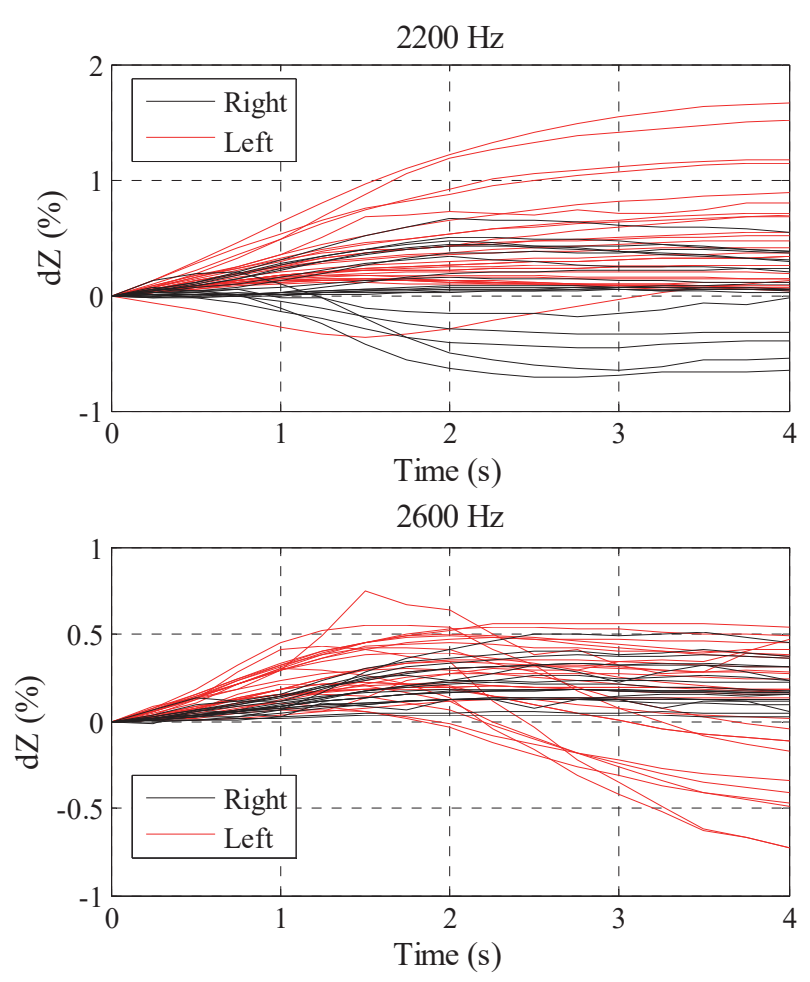

Fig. 7: $\mathrm{dZ}$ values recorded at $2.2 \mathrm{kHz}$ and $2.6 \mathrm{kHz}$ during a single seizure. Seizure onset is at $t=0$. Right and left refers to the site of electrode placement.

The skin on top of the skull was incised in the mid-line and two craniotomies were performed. Electrode arrays [32] were placed on either side of the rat brain. Further details of the anaesthesia and surgical procedure are available in [31]. Thirty electrodes were used for recording on the left hemisphere and sixty on the right. Epilepsy was triggered in the hippocampus, by the injection of 4-AP into the right hemisphere. Two parallel current sources were used, operating at $2.2 \mathrm{kHz}$ and $2.6 \mathrm{kHz}$. The peak injecting current was $50 \mu \mathrm{A}$ and the currents were sourced/sinked through the left/right electrode arrays respectively, with diametrically opposite injection pairs chosen. The BioSemi system was used for simultaneous voltage recording.

The raw recorded data, containing both EEG and EIT data, was split into the two separate components (Figure 6B). Examples of extracted EEG and impedance data (dZ) are shown in Figure 7, over 15 seconds. Seizure onset occurred at 9 seconds. For the seizures shown, the range of impedance changes were from $-0.75 \%$ to $1.6 \%$ at $2.2 \mathrm{kHz}$ and $-0.7 \%$ to $0.6 \%$ at $2.6 \mathrm{kHz}$. Data was recorded over 4 hours and a total of 209 seizures were observed. The maximum impedance changes seen at $2.2 \mathrm{kHz}$ and $2.6 \mathrm{kHz}$ were $6.28 \%$ and $3.91 \%$ respectively. Impedance data gathered from all electrodes during a single seizure is shown in Figure 8. The magnitudes of impedance changes measured were in agreement with existing literature on the subject [24]. 


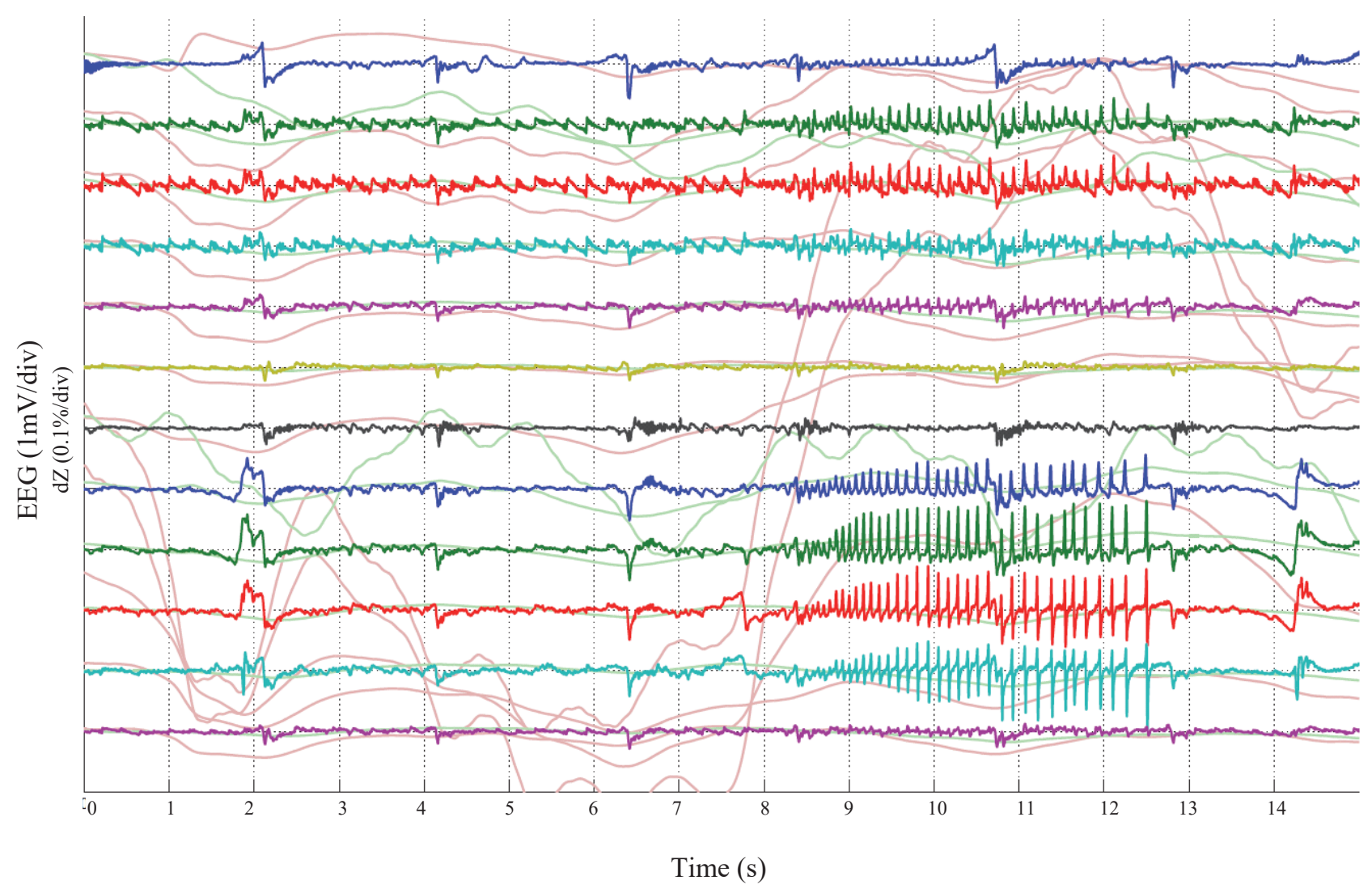

Fig. 8: Example of raw recorded data, recorded from the right hemisphere. Solid colours represent the EEG data. dZ values, from $2.2 \mathrm{kHz}$ injection, are plotted on top of each channel in grey.

\section{Discussion}

An EIT system has been presented that meets the requirements set out for imaging of epileptic seizures. Switching of injection electrodes was eliminated by the use of parallel current sources, operating at different frequencies. The performance of the current source was evaluated, showing excellent stability across time and having an output impedance ( $>10 \mathrm{M} \Omega$ at $10 \mathrm{kHz})$ that is more than sufficient to drive a typical electrode impedance of $<1 \mathrm{k} \Omega$. Tank experiments showed the capability of the system to image static objects, and dynamic events with short durations, using as little as $1 \mathrm{~ms}$ of data. Initial results in a rat model of epilepsy show that EIT data can be used to recorded simultaneously with EEG data, from 90 electrodes at multiple frequencies.

Accurate EIT imaging of epileptic activity will require the use of more than two parallel injections and further experiments are required to gather suitable data sets. Additionally, several separate problems need to be addressed to produce correct images which are beyond the scope of this work, including the provision of real time impedance data, localization of the electrodes and generation of an anatomically accurate mesh of the rat's head. However, the results obtained so far indicate that imaging will be possible using the hardware and approach described in this work.

\section{Acknowledgements}

This work was supported by the Welcome Trust and Medical Research Council.

\section{References}

1. Holder DS. Electrical impedance tomography: methods, history, and applications . Institute of Physics Publishing; 2005.

2. Frerichs I. Electrical impedance tomography (EIT) in applications related to lung and ventilation: a review of experimental and clinical activities. Physiol. Meas. 2000:21(2):R1-R21. http://dx.doi.org/10.1088/0967-3334/21/2/201

3. Hahn G, Just A, Dudykevych T, Frerichs I, Hinz J, Quintel M, Hellige, G. Imaging pathologic pulmonary air and fluid accumulation by functional and absolute EIT. Physiol. Meas. 2006:27(5):S187-S198 http://dx.doi.org/10.1088/0967-3334/27/5/S16

4. Kuen J, Woo EJ, Seo JK. Multi-frequency time-difference complex conductivity imaging of canine and human lungs using the KHU Mark1 EIT system. Physiol. Meas. 2009;30(6):S149S164. http://dx.doi.org/10.1088/0967-3334/30/6/S10

5. Beppu T, Ishiko T, Doi K, Matsuda T, Maeda T, Ishihara K, Ogata K, Ogawa M. A promising new treatment strategy for advanced hepatocellular carcinoma -"multi-ablation therapy" consisting of radio-frequency ablation (RFA), microwave coagulation therapy (MCT) and ethanol injection therapy (EIT). Japanese J. Cancer Chemother. 2012;65(1):23-30. 
6. You F, Shuai W, Shi X, Fu F, Liu R, Dong X. In vivo monitoring by EIT for the pig's bleeding after liver injury. IFMBE Proc. 2009. p. $110-112$.

http://dx.doi.org/10.1007/978-3-642-03879-2_31

7. Halter RJ, Hartov A, Paulsen KD. A broadband high-frequency electrical impedance tomography system for breast imaging. IEEE Trans. Biomed. Eng. 2008:55(2):650-659. http://dx.doi.org/10.1109/TBME.2007.903516

8. Holder DS. Electrical impedance tomography (EIT) of brain function. Brain Topogr. Kluwer Academic Publishers-Human Sciences Press; 1992;5(2):87-93.

9. Bagshaw AP, Liston AD, Bayford RH, Tizzard A, Gibson AP, Tidswell AT, Sparkes M, Dehghani H, Binnie C, Holder S. Electrical impedance tomography of human brain function using reconstruction algorithms based on the finite element method. Neuroimage 2003;20(2):752-764. http://dx.doi.org/10.1016/S1053-8119(03)00301-X

10. Holder D. Electrical impedance tomography in epilepsy. Electron. Eng. Miller Freeman plc; 1998;70(859):69-70.

11. Vongerichten A, Sato dos Santos G, Avery J, Walker M, Holder D. Electrical impedance tomography (EIT) of epileptic seizures in rat models - a potential new tool for diagnosis of seizures. Clin. Neurophysiol. 2014;125(supp. 1):282-283. http://dx.doi.org/10.1016/s1388-2457(14)50924-8

12. Meinardi H, Scott RA, Reis R, On Behalf Of The Ilae Commission on JWASS. The Treatment Gap in Epilepsy: The Current Situation and Ways Forward. Epilepsia. 2001;42(1):136149. http://dx.doi.org/10.1046/j.1528-1157.2001.32800.x

13. Duncan JS. Selecting patients for epilepsy surgery: synthesis of data. Epilepsy Behav. 2011;20(2):230-232. http://dx.doi.org/10.1016/j.yebeh.2010.06.040

14. Aristovich KY, dos Santos GS, Packham BC, Holder DS. A method for reconstructing tomographic images of evoked neural activity with electrical impedance tomography using intracranial planar arrays. Physiol. Meas. 2014;35(6):2095-1109. http://dx.doi.org/10.1088/0967-3334/35/6/1095

15. Vulliemoz S, Lemieux L, Daunizeau J, Michel CM, Duncan JS. The combination of EEG source imaging and EEG-correlated functional MRI to map epileptic networks. Epilepsia. 2010;51(4):491-505. http://dx.doi.org/10.1111/j.1528-1167.2009.02342.x

16. Zhao M, Suh M, Ma H, Perry C, Geneslaw A, Schwartz TH. Focal increases in perfusion and decreases in hemoglobin oxygenation precede seizure onset in spontaneous human epilepsy. Epilepsia. 2007;48(11):2059-2067. http://dx.doi.org/10.1111/j.1528-1167.2007.01229.x

17. Suh M, Bahar S, Mehta AD, Schwartz TH. Temporal dependence in uncoupling of blood volume and oxygenation during interictal epileptiform events in rat neocortex. J. Neurosci. 2005;25(1):6877. http://dx.doi.org/10.1523/JNEUROSCI.2823-04.2005

18. Ahn S, Oh TI, Jun SC, Seo JK, Woo EJ. Validation of weighted frequency-difference EIT using a three-dimensional hemisphere model and phantom. Physiol. Meas. 2011;32(10):1663-1680. http://dx.doi.org/10.1088/0967-3334/32/10/013

19. Bahar S, Suh M, Zhao M, Schwartz TH. Intrinsic optical signal imaging of neocortical seizures: the "epileptic dip". Neuroreport. 2006;27(6):499-503. http://dx.doi.org/10.1097/01.wnr.0000209010.78599.f5
20. McCann H, Ahsan ST, Davidson JL, Robinson RL, Wright P, Pomfrett CJD. A portable instrument for high-speed brain function imaging: FEITER. Annu. Int. Conf. IEEE Eng. Med. Biol. Soc. 2011:7029-7032. http://dx.doi.org/10.1109/IEMBS.2011.6091777

21. Wi H, Sohal H, McEwan AL, Woo EJ, Oh TI. Multi-frequency electrical impedance tomography system with automatic selfcalibration for long-term monitoring. IEEE Trans. Biomed. Circuits Syst. 2014;8(1):119-128. http://dx.doi.org/10.1109/TBCAS.2013.2256785

22. Fabrizi L, Sparkes M, Horesh L, Perez-Juste Abascal JF, McEwan A, Bayford RH, Elwes R, Binnie C, Holder D. Factors limiting the application of electrical impedance tomography for identification of regional conductivity changes using scalp electrodes during epileptic seizures in humans. Physiol. Meas. 2006:27(5):S163S174. http://dx.doi.org/10.1088/0967-3334/27/5/S14

23. Liston A, Bayford R, Holder D. A cable theory based biophysical model of resistance change in crab peripheral nerve and human cerebral cortex during neuronal depolarisation: implications for electrical impedance tomography of fast neural activity in the brain. Med. Biol. Eng. Comput. 2012;50(5):425-437. http://dx.doi.org/10.1007/s11517-012-0901-0

24. Rao, A, Gibson. A.P., Holder DS. EIT images of electrically induced epileptic activity in anaesthetised rabbits. Med. Biol. Eng. Comput. 1997;35(1):327.

25. Fabrizi L, Yerworth R, McEwan A, Gilad O, Bayford R, Holder DS. A method for removing artefacts from continuous EEG recordings during functional electrical impedance tomography for the detection of epileptic seizures. Physiol. Meas. 2010;31(8):S57S72. http://dx.doi.org/10.1088/0967-3334/31/8/S05

26. Granot Y, Ivorra A, Rubinsky B. Frequency-division multiplexing for electrical impedance tomography in biomedical applications. Int. J. Biomed. Imaging. 2007;2007:1-9.

27. Gracia J, Seppa VP, Viik J, Hyttinen J. Multilead measurement system for the time-domain analysis of bioimpedance magnitude. IEEE Trans. Biomed. Eng. 2012;59(8):2273-2280. http://dx.doi.org/10.1109/TBME.2012.2202318

28. McEwan A, Tapson J, van Schaik A, Holder DS. Code-divisionmultiplexed electrical impedance tomography spectroscopy. IEEE Trans. Biomed. Circuits Syst. 2009;3(5):332-338. http://dx.doi.org/10.1109/TBCAS.2009.2032159

29. Tucker AS, Fox RM, Sadleir RJ. Biocompatible, high precision, wideband, improved Howland current source with lead-lag compensation. IEEE Trans. Biomed. Circuits Syst. 2013;7(1):6370. http://dx.doi.org/10.1109/TBCAS.2012.2199114

30. Vauhkonen M, Vadâsz D, Karjalainen PA, Somersalo E, Kaipio JP. Tikhonov regularization and prior information in electrical impedance tomography. IEEE Trans. Med. Imaging . 1998;17(2):285-293. http://dx.doi.org/10.1109/42.700740

31. Vongerichten A. Imaging Physiological and Pathological Activity in the Brain using Electric Impedance Tomography. $\mathrm{PhD}$ thesis, UCL, 2014.

32. Schuettler M, Ordonez JS, Henle C, Oh D, Gilad O, Holder DS. A Flexible 29 Channel Epicortical Electrode Array. IFESS 2008 13th Annu. Int. FES Soc. Conf. 2008. 